\title{
Estudio de asociación genómica para resistencia a Cooperia punctata en bovinos cruzados en el trópico subhúmedo de México
}

Adriana García-Ruíz ${ }^{\text {a }}$

Felipe de Jesús Ruíz-López ${ }^{\text {a }}$

Miguel Alonso-Díaz ${ }^{b}$

Elke Von-Son-de-Fernex ${ }^{b}$

Sara Olazarán-Jenkins ${ }^{c}$

Vicente Eliezer Vega-Murillo ${ }^{\mathrm{d}}$

Maria Eugenia López-Arellano e* $^{*}$

a Instituto Nacional de Investigaciones Forestales Agrícolas y Pecuarias (INIFAP). Centro Nacional de Investigación Disciplinaria en Fisiología y Mejoramiento Animal. Ajuchitlán, Querétaro.

b Universidad Nacional Autónoma de México (UNAM). Centro de Enseñanza, Investigación y Extensión en Ganadería Tropical. Martínez de la Torre, Veracruz. México.

${ }^{c}$ INIFAP. Sitio Experimental las Margaritas, Hueytamalco, Puebla. México.

d INIFAP. Campo Experimental La Posta, Paso del Toro, Veracruz. México.

e INIFAP. Centro Nacional de Investigación Disciplinaria en Salud Animal e Inocuidad. Jiutepec, Morelos. México.

*Autor de correspondencia: mlopez.arellano@gmail.com

\section{Resumen:}

El objetivo del presente estudio fue evaluar la resistencia a la infección natural por Cooperia spp. en bovinos jóvenes cruzados Cebú x Holstein, en trópico. Catorce (14) becerros de cuatro meses de edad fueron tratados con desparasitante y trasladados a potreros naturalmente contaminados con nematodos gastrointestinales bajo condiciones de trópico sub-húmedo. Muestras de heces se colectaron cada siete días durante tres 
meses por individuo, para cuantificar el número de huevos por gramo de heces (HPG) e identificar el género por PCR de punto final. Se colectaron muestras de pelo para llevar a cabo estudios de asociación genómica utilizando la plataforma GeneSeek Genomic Profiler HD-V3 que contiene139,376 marcadores SNP. Los resultados obtenidos indican variación en el número de $\mathrm{HPG}$ por individuo (mínimo $7 \pm 7$ y máximo 4657 \pm 1886 HPG). En los análisis de componentes principales se identificaron diferencias raciales entre los animales con genes de las razas Cebú y Holstein. Los estudios de asociación del genoma completo detectaron 5 haplotipos estadísticamente significativos $(P<0.001)$. El haplotipo del cromosoma 2 incluye cuatro marcadores, el 10 incluye a tres, el 15 incluye a dos, el 23 cuatro y el del cromosoma $\mathrm{X}$ incluye tres. De estas regiones sólo el cromosoma 23 se encontró asociado a resistencia por parásitos, medidos como fenotipo de HPG; los cromosomas restantes identificados no mostraron asociación en ninguno de los individuos en estudio. Se considera que estas regiones podrían ser secuenciadas y probar la expresión de genes para la resistencia contra este nematodo y de otros nematodos del complejo gastrointestinal.

Palabras clave: Marcadores SNP, Asociación genómica, Cooperia spp., Bovinos, Cruzamientos.

Recibido: 30/01/2018

Aceptado: 08/05/2018

La infección por nematodos gastrointestinales (GI) en bovinos jóvenes es uno de los principales problemas de salud que afecta a la ganadería bajo condiciones de pastoreo en clima tropical ${ }^{(1)}$. Cooperia es un parásito GI que tiene un efecto negativo en el crecimiento y la productividad de bovinos, debido a que afecta la función del intestino delgado $^{(2)}$. Se han estudiado diversos mecanismos para combatir la infección por Cooperia, tanto a nivel de fármacos como a nivel de resistencia genética ${ }^{(3)}$. Con la disponibilidad de paneles densos de marcadores de ADN de polimorfismos de un solo nucleótido (SNP), ha sido posible realizar el mapeo de loci de características de interés económico y de esta manera incrementar la presencia de genes deseables en la población. Los haplotipos son regiones del genoma (que comprenden entre 100 y 150 pares de bases) que son heredados de manera conjunta en una población específica y pueden tener efecto significativo sobre los genes que estén localizados dentro de una región del cromosoma ${ }^{(4)}$. Los estudios de asociación en el genoma completo (GWAS) son una herramienta que nos permite identificar estas regiones en el material genético. Recientemente, se han identificado regiones del genoma que indican resistencia a enfermedades y parasitosis ${ }^{(5)}$. 
El objetivo del presente trabajo fue identificar regiones del genoma asociadas a carga parasitaria (número de huevos por gramo de excremento) de Cooperia en ganado bovino cruzado (Cebú x Holstein y viceversa) que se encuentran en pastoreo en una región del trópico en México.

El estudio se llevó a cabo en el Centro de Enseñanza, Investigación y Extensión en Ganadería Tropical (CEIEGT), de la Facultad de Medicina Veterinaria y Zootecnia, de la Universidad Nacional Autónoma de México, localizado en el municipio de Tlapacoyan, Veracruz, a $112 \mathrm{msnm}$. El clima es cálido húmedo, con lluvias todo el año, sin estación seca definida $\left(\mathrm{Af}(\mathrm{m}) \mathrm{w}\right.$ " $(\mathrm{e})^{(6)}$, con temperatura promedio de $23.5 \pm 0.5^{\circ} \mathrm{C}$ y $1,991 \mathrm{~mm} \pm 392 \mathrm{~mm}$ de precipitación media anual y la prevalencia de nematodos GI es mayor al $75 \%$ durante todo el año ${ }^{(7)}$. En el proyecto se incluyeron 14 animales cruzados $(3 / 4$ Cebú x 1/4 Holstein $(n=6)$ y 1/4 Cebú x 3/4 Holstein $(n=8)$ de cuatro meses de edad que se encontraban en pastoreo y que previo a su destete, tanto las crías como las madres, se trataron con Levamisol a dosis de $7.5 \mathrm{mg}$ por vía intramuscular para eliminar posibles contaminaciones con nematodos GI.

Todos los animales se infectaron naturalmente con larvas infectantes $\left(\mathrm{L}_{3}\right)$ de Cooperia punctata (confirmado por PCR de punto final) como género dominante por vía oral, y posterior a la infección se realizaron monitoreos semanales por tres meses del número de huevos por gramo de excremento (HPG). El primer registro de HPG se realizó a los 21 días después de iniciada la infección de los animales en pastoreo y los posteriores se realizaron cada siete días por tres meses. El número de HPG se determinó con la técnica de McMaster. Las muestras se colectaron directamente del recto para evitar contaminación y se procesaron inmediatamente. Se obtuvo el promedio de HPG durante los muestreos, y los resultados se incluyeron en un estudio de casos y controles, considerando como casos a los animales que presentaron más de $200 \mathrm{HPG}^{(8,9)}$. La clasificación que se observa en el Cuadro 1, indica el nivel de infección por el número de HPG de nematodos parásitos. Así mismo, se tomaron muestras de sangre para determinar el porcentaje de volumen celular aglomerado (VCA), tomando como base $24 \%$ como la medición de un individuo no anémico ${ }^{(9)}$. Así mismo, se determinó el VCA con un porcentaje mínimo de $22+1.6$ y máximo de $28+3.3$. Es importante señalar, que el género Cooperia presenta hábitos de histiofagia y no afecta directamente el porcentaje del VCA. Además, este género fue predominante durante los tres meses de estudio. La disminución del VCA se relacionó al estado nutricional de los animales, por ello, se identificaron seis individuos como resilientes. 
Cuadro 1: Selección de becerros resistentes, susceptibles y resilientes a Cooperia punctata con base en el número de huevos por gramo (HPG) y el porcentaje de volumen celular aglomerado (VCA)

\begin{tabular}{cccc}
\hline ID & $\begin{array}{c}\text { HPG } \\
\text { Media } \pm \text { EE }\end{array}$ & $\begin{array}{c}\text { VCA }(\%) \\
\text { Media } \pm \text { EE }\end{array}$ & C \\
\hline 62 & $7 \pm 7$ & $24 \pm 2.2$ & RR \\
58 & $164 \pm 89$ & $24 \pm 1.1$ & RR \\
61 & $200 \pm 107$ & $26 \pm 2.2$ & RR \\
57 & $178 \pm 154$ & $23 \pm 2.8$ & RR \\
55 & $86 \pm 291$ & $22 \pm 0.7$ & Rs \\
56 & $650 \pm 291$ & $28 \pm 1.7$ & Rs \\
51 & $557 \pm 291$ & $24 \pm 3.3$ & Rs \\
67 & $607 \pm 165$ & $22 \pm 1.5$ & Rs \\
65 & $764 \pm 283$ & $23 \pm 1.5$ & Rs \\
63 & $1193 \pm 636$ & $25 \pm 3.3$ & Rs \\
59 & $1221 \pm 558$ & $25 \pm 0.8$ & $\mathrm{SS}$ \\
53 & $1378 \pm 291$ & $26 \pm 2.7$ & SS \\
54 & $3100 \pm 291$ & $22 \pm 2.3$ & SS \\
64 & $4657 \pm 1886$ & $23 \pm 2.1$ & SS \\
\hline X EE & $1055 \pm 1155$ & $24 \pm 2.0$ & \\
ID= identificación por individuo; EE= error estándar C= clasificación de fenotipo por individuo; &
\end{tabular}

A todos los animales se les tomó una muestra de pelo que incluyó el folículo piloso a partir de la cual se obtuvo su ADN. Posteriormente, se genotiparon con la plataforma GeneSeek Genomic Profiler HD-V3 que contiene139,376 SNP. Se realizó el análisis de control de calidad de los genotipos, eliminando los marcadores que presentaran una tasa de llamado un call rate $<0.90$, una frecuencia del alelo menor a $0.05 \mathrm{y}$ aquellos que tuvieran un valor de $P<0.001$ para el equilibrio de Hardy Weinberg. Después del control de calidad, se incluyeron 120,405 SNP en la determinación de 3,054 haplotipos. Para corregir el efecto de origen racial, se realizaron los análisis de componentes principales (PCA) y se incluyeron como factor de ajuste en los análisis de asociación. Los GWAS se realizaron con un modelo de regresión logística, corrigiendo por la prueba de Bonferroni y por la tasa de falsos descubrimientos. Todos los análisis genómicos se realizaron con el software SVS-Golden Helix v-8.6 $6^{(10)}$.

La identificación molecular de los géneros de nematodos GI mostró a Cooperia como el género dominante de la región, no encontrándose otros especímenes durante los tres meses de seguimiento. Respecto al nivel de infección observado por individuo, el Cuadro 1 muestra la media y el error estándar por individuo obtenido durante los tres meses de estudio. El valor mínimo fue de $7 \pm 7$ y el máximo de 4,657 $\pm 1,886$. En los PCA, se pudieron identificar las diferencias de composición racial de los animales incluidos en el estudio (Figura 1), observándose a la izquierda los animales que poseían mayor cantidad de genes de la raza Cebú y a la derecha los que poseían mayor composición Holstein. 
Figura 1: Análisis de componentes principales de los animales incluidos en el estudio y su agrupación por composición racial

- 3/4 HOLSTEIN x 1/4CEBU

- CEBU $3 / 4 \times$ HOLSTEIN 1/4

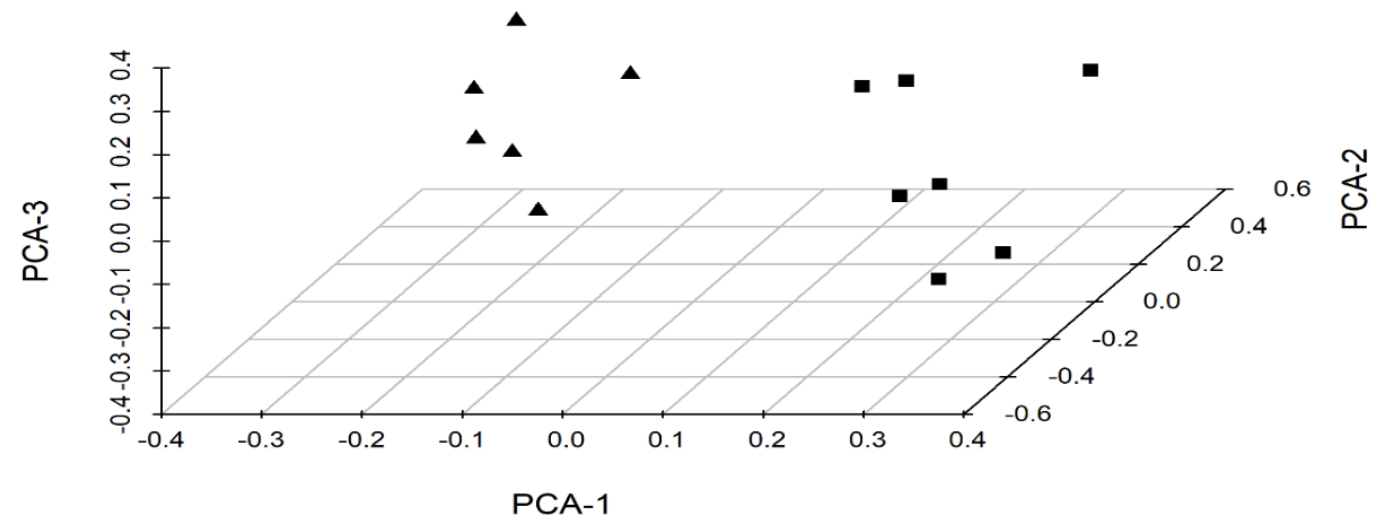

A pesar de que para algunos caracteres o enfermedades existe una predisposición genética a razas, cruzas o poblaciones específicas ${ }^{(11)}$, en este estudio no se encontraron tendencias de casos y controles por cruzamiento asociados a la presencia de Cooperia (Figura 2).

Figura 2: Distribución de casos y controles a Cooperia de los animales incluidos en el estudio

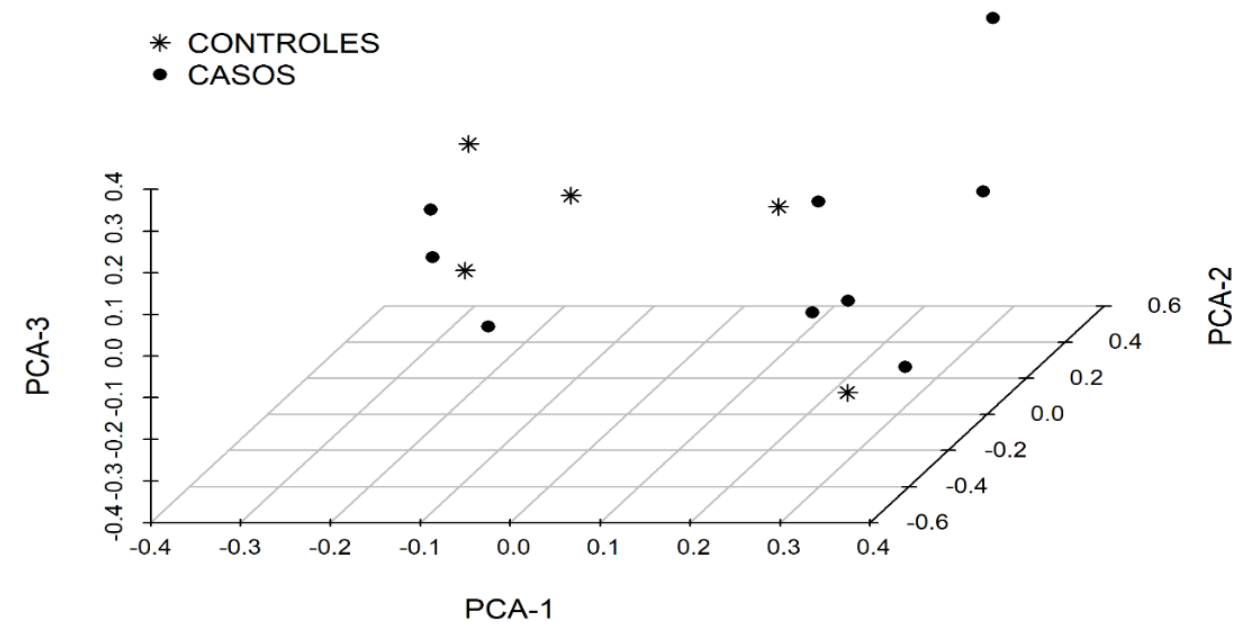

En los GWAS se detectaron 5 haplotipos estadísticamente significativos $(P \leq 0.001$; Figura 3). El haplotipo del cromosoma 2 incluye cuatro marcadores (BTA-55603-no-rs, BovineHD4100001149, BovineHD0200010866, BovineHD0200010867), el del 10 incluye a tres (BovineHD1000009272, ARS-BFGL-NGS-44351, BovineHD10000 
30584), el del 15 incluye a dos (ARS-BFGL-NGS-18481, ARS-BFGL-NGS-103542), el del 23 incluye cuatro (BovineHD2300011368, BovineHD2300011369, BovineHD2300011370, BovineHD2300011371) y el del cromosoma $\mathrm{X}$ incluye tres (BovineHD3000031659, BovineHD3000031667, BovineHD3000031676).

Figura 3: Gráfica tipo Manhattan de análisis de asociación con haplotipos encontrados en el estudio de casos y controles de Cooperia. El cromosoma X se encuentra denotado como cromosoma 30

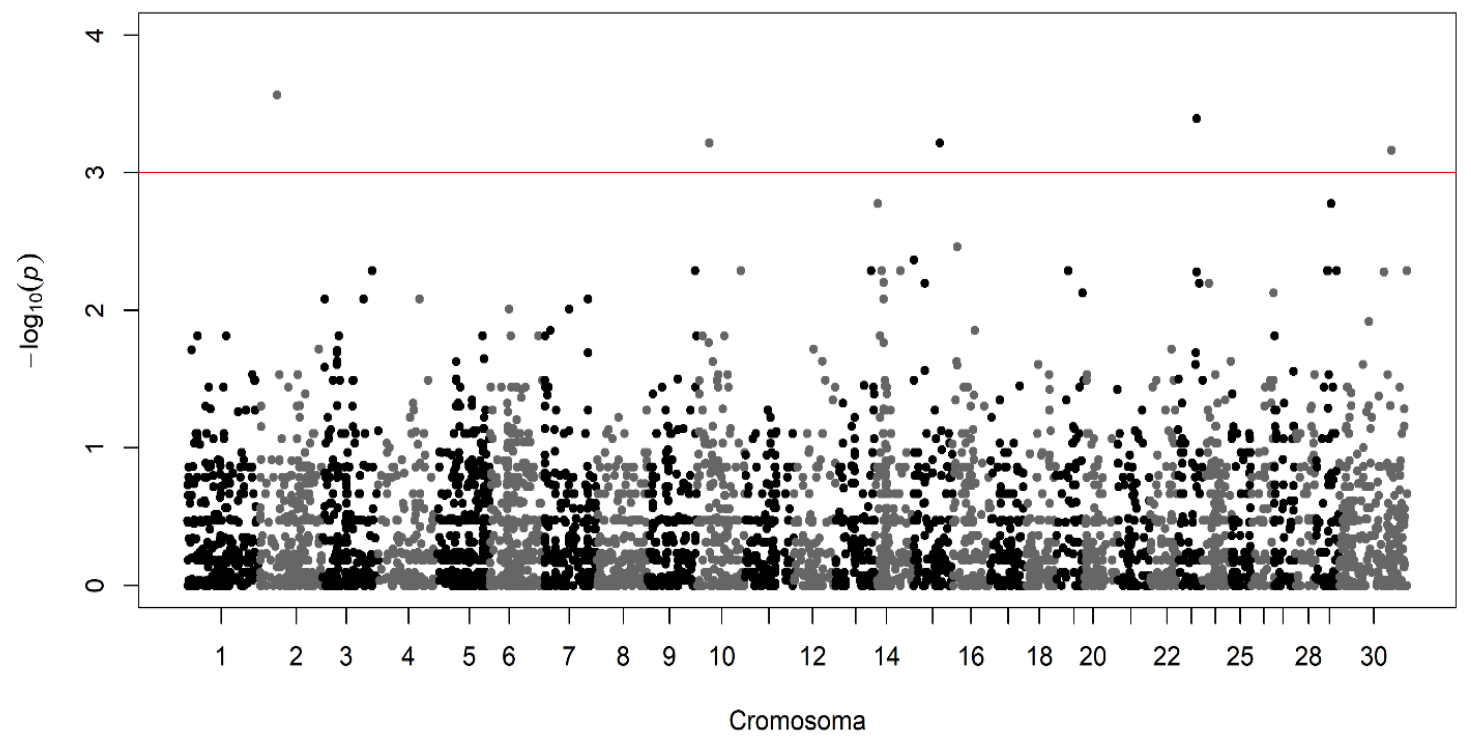

Debido a que los estudios de asociación genómica a resistencia a enfermedades han sido muy limitados, no se tienen muchas anotaciones en el genoma para tomar como referencia. De las regiones significativas que se encontraron en el presente estudio para los cromosomas 2, 10, $15 \mathrm{y} \mathrm{X}$, no se identificaron genes que pudieran estar relacionados con las regiones encontradas (resultados verificados en la base de datos de NCBI) ${ }^{(12)}$. Solo la región que resultó significativa en el cromosoma 23, está asociada con la tasa de infección parasitaria al protozoario Tripanosoma en ganado N'Dama y Boran en África Occidental $^{(13)}$. Debido a que la mayoría de las regiones encontradas no se encuentran asociadas a características de HPG y en específico de Cooperia, se considera que estas regiones pueden ser candidatas para secuenciar y probar la existencia de genes para la resistencia contra este nematodo y de otros del complejo gastrointestinal.

En conclusión, a pesar del bajo número de bovinos jóvenes utilizados en el presente estudio, los resultados encontrados sugieren la existencia de SNP asociados con la resistencia a nematodos, principalmente en el cromosoma 23. Sin embargo, se requiere mayor información para definir dicha relación. El estudio de la interacción hospedero/parásito implica mayor conocimiento en la diversidad de mecanismos inmunes de evasión, y detoxificación antihelmíntica en las nematodosis. La contribución en el conocimiento de factores asociados al genoma del hospedero y a los 
procesos de infección por $C$. punctata implica determinar mecanismos que podrían indicar en un futuro el uso de marcadores asociados a las nematodosis bajo condiciones de pastoreo en trópico.

\section{Agradecimientos}

El presente proyecto fue financiado por el Proyecto Fiscal INIFAP número 16573433030. Se agradece todo el apoyo otorgado por el CEIEGT, FMVZ-UNAM.

\section{Literatura citada:}

1. Borges FA, Almeida GD, Heckler RP, Lemes RT, Onizuka MKV, Borges, DGL. Anthelmintic resistance impact on tropical beef cattle productivity: effect on weight gain of weaned calves. Trop Anim Health Prod 2013;45:723-727.

2. Stromberg BE, Gasbarre LC, Waite A, Bechtol DT, Brown MS, Robinson NA, Newcomb H. Cooperia punctata: Effect on cattle productivity? Vet Parasitol 2013;183(3-4):284-291.

3. Geary TG, Hosking BC, Skuce PJ, von Samson-Himmelstijerna G, Maeder S, Holdsworth P, Pomroy W, Vercruysse J. World Association for the Advancement of Veterinary Parasitology (W.A.A.V.P.) Guideline: Anthelmintic combination products targeting nematode infections of ruminants and horses. Vet Parasitol 2012;190:306-316.

4. Fritz S, Capitan A, Djari A, Rodriguez SC, Barbat A. Detection of haplotypes associated with prenatal death in dairy cattle and identification of deleterious mutations in GART, SHBG and SLC37A2. PLoS ONE 2013;8(6):e65550.

5. Coppieters W, Mes TH, Druet T, Farnir F, Tamma N, Schrooten C, Ploeger HW. Mapping QTL influencing gastrointestinal nematode burden in Dutch HolsteinFriesian dairy cattle. BMC Genomics 2009;10(1):96.

6. García E. Modificaciones al Sistema de Clasificación Climática de Köppen. 3a. ed. Instituto de Geografía. UNAM. México. D.F. 1981.

7. Alonso-Díaz MA, Arnaud-Ochoa RA, Becerra-Nava R, Torres-Acosta JFJ, Rodriguez-Vivas RI, Quiroz-Romero RH. Frequency of cattle farms with ivermectin resistant gastrointestinal nematodes in Veracruz, Mexico. Vet Parasitol 2015;212:439-443. 
8. Coles GC, Bauer C, Borgsteede FHM, Geerts S, Klei TR, Taylor MA, Waller P, World Association for the Advancement of Veterinary Parasitology (W.A.A.V.P.) methods for the detection of anthelmintic resistance in nematodes of veterinary importance. Vet Parasitol 1992;44:35-44.

9. Encalada-Mena L, Tuyub-Solis H, Liébano-Hernández E, Ramirez-Vargas G, Mendoza-de-Gives P, Aguilar-Marcelino L, López-Arellano ME. Phenotype and genotype traits of gastrointestinal nematodes resistant to benzimidazole in infected calves from tropical regions of Campeche State, Mexico. Vet Parasitol 2014;205:246-254.

10. Golden Helix Inc. SNP \& Variation Suite Manual Version 8.6.0 Copyright (C 20002016 Golden Helix, Inc. 2016.

11. Zhang H, Wang Z, Wang S, Li H. Progress of genome wide association study in domestic animals. J Anim Sci Biotech 2012;3:26.

12. National Center for Biotechnology Information. https://www.ncbi.nml.nih.gov Accessed Jul 15, 2017.

13. Gelhaus A, Horstmann R, Teale A. Mapping of quantitative trait loci controlling trypanotolerance in a cross of tolerant West African N'Dama and susceptible East African Boran cattle. Proc Nat Acad Sci USA 2003;100(13):74. 